\title{
Análisis y estrategias en la aplicación de la evaluación formativa y compartida en magisterio
}

\section{Analysis and strategies in the implementation of formative and shared assessment in teacher education}

Maria Laura Angelini*

\section{RESUMEN}

Este trabajo analiza la aplicación de la evaluación formativa y compartida en la formación del profesorado en Maestro especialista en lengua inglesa. Se realiza un repaso de los fundamentos teóricos que avalan esta modalidad de evaluación y se lleva a cabo un estudio cualitativo y cuantitativo con una población de futuros maestros de inglés correspondiente a tres cursos consecutivos. Se detalla el procedimiento para la obtención de los datos que dan cuerpo a la presente investigación; se efectúa el análisis de los mismos, de los que se extraen las conclusiones. Se ha podido comprobar que la evaluación formativa y compartida ejerce un papel fundamental en el compromiso del alumnado con su propia formación, desarrolla su autonomía, propicia un seguimiento cercano y diferenciado de él, desarrolla competencias lingüísticas y transversales como también permite un mejor desempeño académico. Asimismo, se han identificado ciertas dificultades que están ligadas a una posible inseguridad por parte del alumnado frente a la propuesta de autoevaluación y coevaluación, así como respecto a la coordinación del trabajo en equipo y el tiempo requerido para llevar a cabo los proyectos.

Palabras clave: evaluación formativa, evaluación compartida, formación de maestros, autoevaluación, competencias lingüísticas, competencias profesionales

\section{ABSTRACT}

In this research work, we analyze the implementation of formative and shared assessment in our teacher training degree specialized in English as a Foreign Language. We present a detailed review of the literature and the methodology followed, as well as the results of a qualitative and quantitative study conducted with a group of students -future teachers of Englishduring three consecutive courses. The procedure for data gathering is specified, along with the data analysis from which conclusions are drawn. Results reveal that this type of assessment plays an important role in promoting the learners' commitment to their own learning process. It also promotes the development of autonomy, as well as closer support and differentiated instruction from their teachers. Learners also develop linguistic and professional skills, and achieve better academic outcomes. Some weaknesses in the implementation of formative and shared assessment are also depicted. These weaknesses are associated to a possible lack of self-confidence on the part of the learners to conduct their self-assessment and peerassessment. Some flaws are also found in teamwork coordination and the amount of time necessary to carry out the projects.

Key words: formative assessment, shared assessment, teacher training, self-assessment, linguistic skills, professional competencies

* Departamento de Lengua Inglesa. Universidad Católica de Valencia, España; marialaura. angelini@ucv.es 
El proceso de convergencia regulado por Espacio Europeo de Educación Superior (EEES) provee directrices que deben acatar las universidades europeas en materia de organización y adaptación metodológica. Se insta a las instituciones a aplicar metodologías centradas en el alumno, o metodologías activas de aprendizaje, a la vez que a diseñar sistemas de evaluación más consecuentes con los métodos empleados (Bordas y Cabrera, 2001; Zabalza Beraza, 2003; Biggs, 2005; Mérida Serrano, 2006). Una población estudiantil autorregulada que desarrolle estrategias metacognitivas y cognitivas puede contribuir a un mejor rendimiento académico (Knight, 2005; Lamas Rojas, 2008; Weinstein, Acee y Jung, 2011; y Gargallo López et al., 2014). Por lo tanto, es necesario que las instituciones educativas fomenten la autonomía del alumnado para que sea capaz de gestionar su propio tiempo, de asimilar la información para convertirla en conocimiento y poder aplicarla a nuevas situaciones (Kolb y Kolb, 2005a, 2005b). Para ello se considera primordial ejercer un cambio en los modelos de evaluación tradicionalmente utilizados. Según Perassi (2008), es necesario reivindicar el carácter formativo de la evaluación y su capacidad retroalimentadora para corregir rumbos de acción. Se advierte una creciente preocupación por alejarse de las concepciones de evaluación que se orientan solo a resultados y acercarse a la idea de evaluación del proceso y la reflexión de lo acontecido (Martinic et al., 2014). El objeto de reflexión gira en torno al contenido aprendido, al proceso de enseñanza-aprendizaje, a las metodologías y estrategias de enseñanza y de aprendizaje, entre otros.

Por lo tanto, en este trabajo se presentan los resultados de la aplicación de la evaluación formativa y compartida en la formación de maestros de inglés de la Facultad de Psicología, Magisterio y Ciencias de la Educación de la Universidad Católica de Valencia "San Vicente Mártir". El objetivo de este estudio es analizar los resultados de la evaluación formativa y compartida en $3^{\circ}$ ańo de la carrera de Magisterio, con especialidad en lengua inglesa, y presentar una serie de estrategias metodológicas para su aplicación. Resulta oportuno hacer algunas aclaraciones terminológicas al respecto. Cuando en este trabajo se menciona la participación del estudiantado en su propia evaluación, nos referimos a alumnos que evalúan el desempeño de sí mismos durante el 
proceso de enseñanza-aprendizaje. Entre las diversas investigaciones con esta temática podemos destacar referencias sobre la autoevaluación que aluden a la evaluación que el alumnado hace de sí en los estudios de Freire (1990); Andreu Andrés y García Casas (2006); López Pastor (2009); Martínez Muñoz, Santos Pastor y Sicilia (2006); Watts y García Carbonell (2006); Watts, García Carbonell y Martínez Alzamora (2006). Como bien afirman López Pastor, González Pascual y Barba Martín (2005); Rodríguez Gómez, Ibarra Sáiz y García Jiménez (2013), el término de autoevaluación puede también aplicarse a la autoevaluación del profesorado. La coevaluación, por su parte, puede referirse a la evaluación entre pares de alumnos y a la evaluación entre profesores. Aquí nos referiremos a la autoevaluación como la valoración que el estudiantado hace de sí, mientras que la coevaluación será la evaluación entre pares de estudiantes.

Con respecto al término evaluación compartida, nos referimos al seguimiento del estudiantado por parte del profesorado, que es de carácter dialógico y personalizado sobre su evolución en la consecución de las tareas encomendadas. Hay sesiones individuales y grupales, ya que se trabaja en equipos de no más de cinco alumnos. Somervell (1993) y más tarde Cebrián de la Serna, Serrano Ángulo y Ruiz Torres (2014) relacionan estas prácticas con lo que denominan evaluación colaborativa y cooperativa.

Puede afirmarse que estos modelos de evaluación responden a la denominada evaluación democrática. Como bien sostiene López Pastor (2006), un modo de vida democrático requiere aprendizaje, hábitos, competencias y responsabilidades. En nuestro caso, y teniendo en cuenta la formación de educadores, reafirmamos nuestra convicción de hacer un ejercicio altamente responsable de los procesos de evaluación que partan desde la universidad y que repercutan en la calidad de formación personal y profesional de cada maestro.

\section{ESTADO DE LA CUESTIÓN}

La innovación pedagógica en educación superior, como en otros niveles de enseñanza, debe incorporar modelos de evaluación que aseguren la efectividad del proceso de enseñanza-aprendizaje. Po- 
demos afirmar que el modelo de evaluación es el que articula todo el proceso de enseńanza-aprendizaje. Asimismo, podemos acceder a numerosos estudios que critican negativamente los métodos tradicionales de evaluación, cuyo principal objetivo es calificar el resultado final de un proceso de aprendizaje mediante un examen final (González Pérez, 2001; Dochy, Segers y Dierick, 2002; Rodríguez Mena García y García Montero, 2003; Bretones Román, 2003; Zabalza Beraza, 2002, 2003; López Pastor, 2004; Biggs, 2005; Boud y Falchikov, 2007; Pérez Pueyo et al., 2008; Zaragoza Casterad, 2009, entre otros). Sin embargo, encontramos cada vez más literatura especializada que posiciona a la evaluación formativa como la alternativa a los métodos tradicionales de evaluación. Podemos encontrar numerosas referencias sobre evaluación formativa en educación superior (Bordas y Cabrera, 2001; Zabalza Beraza, 2002; Álvarez Méndez, 2003; López Pastor, 2004; Bonsón y Benito, 2005; Watts y García Carbonell, 2006, entre otros) y numerosos estudios que aluden a la evaluación para el aprendizaje como eje que articula el proceso de enseñanza-aprendizaje (Biggs, 1999; Brown y Glasner, 2003; Delgado García et al., 2005; Klenowski, 2005; Marshall y Drummond, 2006; Villardón Gallego, 2006, entre otros).

Podemos identificar a un estudiante autorregulado si sabe manejar con soltura estrategias de aprendizaje que incluyan elementos de motivación intrínseca (querer aprender), metacognitivos (tomar decisiones y evaluarlas) y cognitivos (poder llevar a cabo el proceso de evaluación) (Reynolds, Livingston y Willson, 2006; Hannafin, 2012 en Gargallo López et al., 2014: 21-59; Hannafin et al., 2014). En esta línea, Rice (2014: 117-118) delimita el constructo evaluación como aprendizaje a través del diseño de herramientas de evaluación centradas en el aprendizaje. El autor identifica siete elementos que aseguran el éxito en la evaluación como aprendizaje: 1) detectar las ganas de aprender; 2) hacerse cargo de esa necesidad de aprender; 3 ) aprender haciendo (práctica-error, experimentación, repetición); 4) aprender de la devolución/feedback (compensación, crítica, observación de resultados); 5) dar sentido a lo aprendido; 6) transmisión de lo aprendido a otros (enseńanza); 7) realización de juicios de valor respecto al propio trabajo, el trabajo de otros, la propia evaluación y la eva- 
luación a pares. Numerosos estudios empíricos demuestran que la evaluación formativa y compartida incide de forma positiva en la implicación del alumnado en su propio aprendizaje, como también contribuye a desarrollar el análisis crítico, la autocrítica y la autonomía del estudiantado (Watts, García Carbonell y Martínez Alzamora, 2006; Watts y Carbonell, 2006; Álvarez Valdivia, 2008; López Pastor, 2009; Martínez Rizo, 2009, Fernández March, 2010; Morales, 2010; Castejón Oliva et al., 2011; López Pastor et al., 2012). Al mismo tiempo, este tipo de evaluación tiene un impacto cualitativo al mejorar y optimizar los procesos de enseñanza-aprendizaje que parten desde la universidad (Dochy, Segers y Dierick, 2002; Brown y Glasner, 2003; De la Fuente y Justicia, 2003; De Miguel Díaz, 2006; López Pastor, 2009; Martínez, Martín y Capllonch, 2009; Contreras, 2011; López Pastor et al., 2012; entre otros).

En lo referido a la formación de maestros de inglés, existen varios trabajos que abogan en favor de la evaluación formativa y compartida. Yi'An (2001), Wen-ming (2003), Zhi-qiang, (2003), Arias y Maturana (2005), Zou y Cai, (2006); Na (2007), López (2010), Arias, Maturana y Restrepo (2012), Restrepo y Nelson (2013), entre otros, coinciden en que existen fundamentos pedagógicos que justifican la aplicación de la evaluación formativa y compartida. Los autores afirman que este tipo de evaluación potencia positivamente el aprendizaje del inglés, debido al grado de responsabilidad que asume el alumnado, su rol activo al tener que evaluarse (autoevaluación) y evaluar al resto del estudiantado (evaluación de pares). Los autores, a su vez, destacan que la evaluación formativa y compartida resulta de utilidad para detectar errores a tiempo. Esto incide directamente en la mejora de los conocimientos del inglés y en los procesos de aprendizaje de la población estudiantil.

\section{METODOLOGÍA}

El presente estudio se lleva a cabo en el Departamento de Lengua Inglesa de la Facultad de Psicología, Magisterio y Ciencias de la Educación, de la Universidad Católica de Valencia "San Vicente Mártir", en la asignatura, Teaching EFL and Children's Literature; 
esta es cuatrimestral de seis créditos, y se imparte de septiembre a diciembre con una carga horaria de cuatro horas y media presenciales a la semana. Está contemplada en el plan de estudios de maestro de preescolar y primaria con especialidad en lengua inglesa. Los alumnos, cuyas edades rondan los 21 ańos de media, se encuentran en $3^{\circ}$ ańo de la carrera. La propuesta docente y la aplicación de la evaluación formativa y compartida se viene desarrollando durante tres cursos consecutivos y el total de la muestra recogida pertenece a los periodos 2012-2013, 2013-2014 y 2014-2015 con un total de 204 alumnos.

Para analizar y valorar el modelo de evaluación propuesto se lleva a cabo un estudio cualitativo y otro cuantitativo. Para el estudio cualitativo, los datos se recogen mediante entrevistas personalizadas por equipos e individuales; una pregunta abierta acerca de las percepciones del alumnado respecto a la evaluación de la asignatura postratamiento; para el estudio cuantitativo, mediante una parrilla que recoge las calificaciones de la autoevaluación y evaluación de pares; una rúbrica que sigue el profesor para calificar las exposiciones orales y que es de conocimiento de los alumnos desde el inicio del curso.

Para el estudio cualitativo, los datos recogidos siguen un sistema de categorización que facilita su análisis. Se sigue el protocolo de la teoría fundamentada de Charmaz (2006), basada en la de Glaser y Strauss (1967), a través del Método Comparativo Constante. Las respuestas se acotan por temas y se codifican en categorías y subcategorías hasta la saturación de datos, y luego estos se someten a análisis para la obtención de resultados. Las muestras obtenidas se analizan con el paquete informático para el análisis de datos cualitativos Atlas.ti, versión 5.2 (Casasempere, 2007).

Para el estudio cuantitativo, se pretende encontrar correlaciones entre las calificaciones del alumnado y del profesor, por lo que se utiliza el coeficiente de correlación de Spearman con el soporte informático Excel versión 2010 para determinar una diferencia de rangos. Se sigue el modelo presentado por López Pastor (2008) por su claridad en el tratamiento. Para garantizar la validez y la confiabilidad del estudio, se han asignado las calificaciones del alumnado antes de revisar las autoevaluaciones. Enseguida presentamos la metodología llevada a cabo: 
ANÁLISIS Y ESTRATEGIAS EN LA APLICACIÓN DE LA EVALUACIÓN FORMATIVA Y COMPARTIDA...

FIGURA 1. Metodología empleada en la asignatura Teaching EFL and Children's Literature

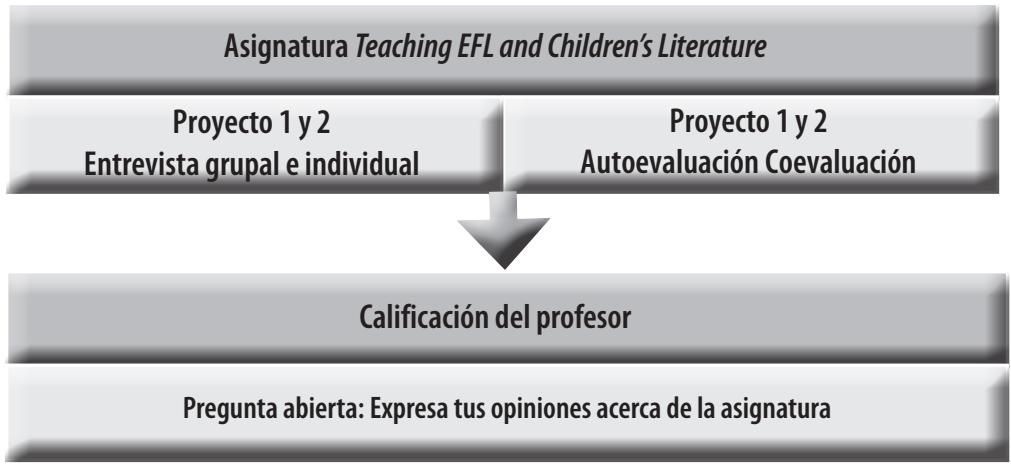

Para la asignatura Teaching EFL and Children's Literature, como estrategia metodológica, se crean equipos de trabajo de no más de cinco estudiantes. Cada integrante asume una responsabilidad dentro del equipo: líder, secretario, asesor/es, monitor lingüístico. Intentamos que este último tenga mejor dominio de la lengua inglesa para garantizar el correcto uso de la misma durante las actividades. Se cuenta con una media de 30 a 35 alumnos por clase. Se trabaja con una amplia variedad de material literario para la enseńanza del inglés en preescolar y $1^{\circ}$ de primaria. El estudiantado analiza el material y realiza adaptaciones en el nivel lingüístico. Se aplican técnicas de enseñanza basadas en la secuencia didáctica: pre-teaching/actual teaching/post-teaching.

Cada equipo selecciona un cuento para contar y el nivel de enseñanza al que va dirigido. Se realizan entrevistas grupales e individuales con el profesor durante los cuatro meses de duración de la asignatura, en las que el maestro recoge las percepciones del alumnado respecto al trabajo en equipo, la dificultad de las actividades, el grado de participación individual en el equipo y la participación de los otros miembros mediante la toma de notas. Este seguimiento permite aclarar cuestiones relativas a los proyectos, a la didáctica del inglés y su aplicación en la enseñanza en los niveles de preescolar y primaria.

Se lleva a cabo la exposición del proyecto 1: contar el cuento seleccionado siguiendo la secuencia pre-teaching/actual teaching/ post-teaching. Los miembros del equipo que expone llevan a cabo 
la autoevaluación y la coevaluación, preferiblemente realizadas fuera de clase. Se debe efectuar de forma anónima para garantizar que no haya malos entendidos entre los participantes. Recordemos que en este tipo de evaluación, las puntuaciones en escala de $0-10$, siendo 10 sobresaliente y 0 insuficiente, pueden fluctuar y, por tanto, no todos los miembros del equipo recibirán la misma puntuación. En todos los casos, se debe justificar las puntuaciones otorgadas por escrito. Al acabar la exposición, el profesor debe haber rellenado su rúbrica atendiendo a aspectos tales como la organización, el uso de la lengua, la secuencia didáctica, las transiciones entre actividades y el manejo del aula. El profesor también debe contar con los informes de autoevaluación y coevaluación, los cuales se revisan posteriormente a la calificación otorgada por el profesor.

El proyecto 2, también sigue el mismo procedimiento pero con material destinado a alumnos del $2^{\circ}$ y $3^{\circ}$ de primaria.

Al finalizar los dos proyectos, se solía evaluar al alumnado mediante un examen global escrito con preguntas de opción múltiple y de desarrollo, que recogía aspectos conceptuales y prácticos del análisis literario, el desarrollo evolutivo del niño y la didáctica del inglés en el aula de infantil y primaria durante los dos primeros años que corresponden al estudio. Tras reflexionar sobre la relevancia de un tipo de examen rígido y el carácter práctico de la asignatura, se decide sustituir al examen final por una herramienta que aportará mayor grado de implicación al alumnado: el portafolio digital.

Es decir, observamos que el examen final no cumplía con dos de los propósitos principales que, según Reynolds, Livingston y Willson (2006), son: 1. mejorar el proceso de aprendizaje de los estudiantes y 2. alinear la evaluación con el proceso de enseñanza. Por eso, en el curso lectivo 2014-2015, el portafolio digital se transforma en una herramienta de medición de aprendizaje con el fin de brindar al alumnado mayor interacción entre pares vía digital y mayor visibilidad "profesional" al exponer sus propias inquietudes públicamente. Un futuro estudio analizará las ventajas y los aspectos mejorables respecto a la utilización del portafolio digital individual como herramienta de formación docente. Por tanto, para el análisis de resultados se omite la información que 
proviene del examen global en los dos cursos anteriores y la pertinente al portafolio digital. Para finalizar, el alumnado responde por escrito a la pregunta abierta postratamiento: Expresa tus opiniones acerca de la asignatura. Una vez recogidas las percepciones de la plataforma de la universidad se procede a su análisis.

La modalidad de evaluación responde, por un lado, a una vía mixta entre evaluación formativa y compartida (entrevistas con el profesor de manera individual y grupal, autoevaluación y coevaluación); para las exposiciones orales de los proyectos se siguen unos criterios que responden a aspectos como el uso de la lengua inglesa (ritmo, entonación, pronunciación, corrección en uso de expresiones y vocabulario); el manejo de la clase (distribución de elementos dentro del aula y control de comportamiento); puesta en escena (una exposición natural, no memorizada, uso de cambios de tonos de voz, expresividad); contenido (adaptación del contenido adecuada a las edades de los infantes, contenido adaptado al tiempo estipulado); organización (seguimiento claro de la secuencia didáctica, uso de transiciones entre actividades que atraigan el interés de los niños), según los criterios adaptados de Matthews y Marino (1990). El cuadro 1 recoge los porcentajes establecidos para cada actividad.

CUADRO 1. Modalidad de evaluación y porcentajes

\begin{tabular}{|c|c|c|}
\hline Evaluación & Porcentajes & Núm. \\
\hline Proyecto 1. Coevaluación y autoevaluación & 10 & \multirow{8}{*}{204} \\
\hline Proyecto 1. Evaluación del profesor (asistencia, entrevistas individuales y grupales) & 10 & \\
\hline Proyecto 2. Coevaluación y autoevaluación & 10 & \\
\hline Proyecto 2. Evaluación del profesor (asistencia, entrevistas individuales y grupales) & 10 & \\
\hline Exposición del proyecto 1-Calificación del profesor & 20 & \\
\hline Exposición del proyecto 2-Calificación del profesor & 20 & \\
\hline (Examen) -Portafolio & 20 & \\
\hline Total asignatura: Suma & 100 & \\
\hline
\end{tabular}

El alumnado que no puede acudir a clases de forma regular no está contemplado en este trabajo de investigación.

A continuación expondremos los resultados de los estudios realizados contando con la muestra total perteneciente solo a 
alumnos regulares (204) que llevan a cabo la evaluación formativa y compartida. Tanto estudiantes como profesor atienden a los siguientes criterios: participación activa; intercambio de ideas; toma de responsabilidades; colaboración y flexibilidad; uso de la lengua inglesa y aspectos mejorables de la propuesta.

De tal manera, solo nos centraremos para el proyecto 1 en autoevaluación y coevaluación, y autoevaluación y evaluación del profesor (asistencia, entrevistas individuales y grupales), y para el proyecto 2 , en autoevaluación y coevaluación, y evaluación del profesor (asistencia, entrevistas individuales y grupales). De esta manera, tanto alumnos como profesor siguen los mismos criterios de evaluación y la misma carga porcentual en cada caso.

\section{ANÁLISIS DE RESULTADOS}

Los resultados de los estudios arrojan tres categorías de análisis:

1) la evaluación formativa y compartida en la enseñanza del inglés y la literatura;

2) resultados de aprendizaje;

3) aspectos mejorables.

\section{La evaluación formativa y compartida en la enseñanza del inglés y la literatura}

La propuesta de evaluación formativa y compartida resulta innovadora para la gran mayoría del alumnado, que manifiesta entusiasmo respecto al trabajo en equipo.

Personalmente, me ha gustado colaborar en un proyecto en común. Creo que en la medida que trabajábamos en el proyecto, leyendo y adaptando el cuento, mi interés por la asignatura fue creciendo y fui tomando sentido a la importancia de la didáctica (en ningún momento dice qué es esto).

Agradezco el esfuerzo de todo mi equipo y creo que esta forma de trabajar es necesaria para aprender todos de todos.

Algunos reconocen que el interés por llevar a cabo el proyecto se potencia debido al apoyo y esmero del resto de participantes. 
Esta manera de evaluar ha servido, a su vez, para acercar posturas respecto a la importancia del manejo de técnicas y métodos de aula.

He aprendido mucho sobre la atención a las distintas destrezas lingüísticas gracias a las charlas con la profesora, quien nos orientaba en el seguimiento de la secuencia didáctica. Disfruté mucho con la preparación de material y juegos.

Me ha resultado complejo planificar toda una clase en inglés y he aprendido de mis compańeros y de sus ideas para poder contar el cuento de manera atractiva a alumnos de primaria... He aprendido a no perder de vista mi función como formador en inglés; y por lo tanto, a la vez que desarrollaba una actividad, debía prestar atención al uso de la lengua y al vocabulario. El monitor lingüístico ha sido muy útil en todo el proceso.

Respecto al nivel de inglés, en algunos casos se percibe una actitud positiva por la confianza que inspira contar con el monitor lingüístico, quien no solo regula la lengua utilizada sino que ayuda a los miembros del equipo mediante explicaciones.

La evaluación formativa y compartida, para algunos, responde a una vía más justa de evaluación, ya que pueden participar de su nota final. Según afirman, esta propuesta arroja transparencia respecto a los propios logros y los del equipo y permite al alumnado identificar las debilidades que deben reforzar: "La evaluación me ha parecido justa porque pude ser honesta conmigo misma y autoevaluarme. Me di cuenta de mis errores y pude valorar el esfuerzo de mis compañeros".

Si bien hay quien disiente con esta propuesta de evaluación por considerarla inadecuada, para la mayoría puede entenderse como un entrenamiento de futuros docentes para aprender a autoevaluarse y evaluar a los demás. Muchos manifiestan satisfacción a la hora de dialogar las propuestas y tomar decisiones: "Considero que la evaluación es menester del profesor y no me veo capacitada para evaluar a los demás".

Algunos aluden ciertas dificultades al trabajar de manera cooperativa por no llegar fácilmente a acuerdos pero, en cualquier caso, han podido llevar a cabo los proyectos, afianzar las rela- 
ciones interpersonales y asumir responsabilidades dentro de su comunidad de aprendizaje.

Resulta difícil hacerse entender cuando cada uno tiene ideas distintas respecto al proyecto final. Hemos debido adaptarnos a otras formas de pensar y el proyecto lo hemos hecho con los aportes de casi todos por igual.

La mejor forma de organizarnos ha sido asumir roles específicos ya que al principio íbamos perdidos. De este modo, todos hemos trabajado y respetado las ideas de los demás.

Para esta asignatura se trabaja en equipo y cada participante asume una responsabilidad dentro del mismo. Se insiste en desempeñar un papel en función de los diferentes estilos de trabajo y de aprendizaje. Si bien el profesorado incide poco en las decisiones de cada equipo en este aspecto, es cierto que en caso de dificultades a la hora de determinar una responsabilidad específica o conflicto interno en algún equipo es el profesor o profesora quien llama al consenso y colabora para que las tareas puedan desarrollarse. Esto ha permitido a todos encontrar su lugar dentro del equipo y realizar aportes valiosos a los proyectos: búsqueda bibliográfica, adaptaciones de historias, traducciones, utilización de recursos tecnológicos, analizar críticamente las historias, los guiones y la puesta en escena, evaluar el desempeńo propio y el de los demás en pos de un bien común.

Como se ha señalado en el apartado de metodología, existen correlaciones entre los valores asignados a la autocalificación de alumnado (ACA), la coevaluación (COE) y la calificación del profesor (CPR). El coeficiente de correlación de Spearman para una muestra de $\mathrm{N}=204$ y un $\mathrm{P}>0.000$. El cuadro 2 recoge los resultados obtenidos.

CUADRO 2. Correlaciones entre autocalificación (ACA), coevaluación (COE) y calificación del profesor (CPR)

\begin{tabular}{|l|c|c|c|}
\hline & ACA & COE & CPR \\
\hline ACA & 1 & & \\
\hline COE & 0.88675 & 1 & \\
\hline CPR & 0.86334 & 0.73211 & 1 \\
\hline
\end{tabular}


Se observa una correlación alta entre COE y ACA (0.88675) que nos indica la existencia de un acuerdo entre la participación y los aportes individuales al equipo, así como las valoraciones entre sus miembros. También se dan coeficientes de correlación altos entre CPR y ACA (0.86334) y CPR y COE (0.73211). Podemos inferir que las autocalificaciones y las calificaciones entre pares (coevaluación) y las del profesor son bastante confiables. Un segundo nivel de análisis profundiza sobre estos resultados. El cuadro 3 recoge las diferencias entre ACA, COE y CPR, el grado y la dispersión de las diferencias.

Como se comprueba en el cuadro 3, las calificaciones de ACA coinciden exactamente con las CPR en 21 casos. Se puede comprobar que 129 casos presentan calificaciones inferiores por parte del profesor, siendo la diferencia en 115 casos de 0.5 puntos, en 13 casos de un punto y en un caso de dos puntos. Esto nos indica, por un lado, que las diferencias son muy pequeńas y que el proceso resulta confiable.

CUADR0 3. Diferencias y dispersión entre autocalificación (ACA), coevaluación (COE) y calificación del profesor (CPR)

\begin{tabular}{|l|c|c|c|c|}
\hline & $N 204$ & \multicolumn{3}{|c|}{ Diferencia escala 1-10 } \\
\hline & & 0.5 & 1 & 1.5 \\
\hline$A C A=C P R$ & 21 casos & - & - & - \\
\hline$C P R>A C A$ & 3 casos & - & - & - \\
\hline$C P R<A C A$ & $129 \operatorname{cas} 05$ & 115 casos & 13 casos & 1 caso (de 2 puntos) \\
\hline$A C A=C O E$ & $37 \operatorname{cas} 05$ & - & - & - \\
\hline$C O E=C P R$ & $18 \operatorname{cas} 05$ & - & - & - \\
\hline$C O E>A C A$ & $37 \operatorname{cas} 05$ & $31 \operatorname{cas} 05$ & 6 casos & - \\
\hline$C O E<A C A$ & $19 \operatorname{cas} 05$ & $14 \operatorname{cas} 05$ & 5 casos & - \\
\hline
\end{tabular}

Respecto a las diferencias poco significativas que se desprenden de este estudio entre CPR y ACA, podemos afirmar que parte del alumnado utiliza criterios relativamente más generosos a la hora de autocalificarse, y el profesor denota más rigurosidad en sus calificaciones. Se pueden extraer varias conclusiones al respecto. El estudiantado puede percibir mayor dificultad para llevar a cabo las tareas debido a la barrera lingüística que supone el inglés. 
Si bien este tipo de alumno puede haberse esforzado en todos los aspectos para cumplir con los requisitos de la asignatura, carece de cierta madurez lingüística necesaria para desenvolverse con corrección y soltura. Esto lo observa fácilmente el profesor, quien puntúa más bajo en este aspecto. Llama la atención que las diferencias en las puntuaciones se den en 129 casos, 13 casos con una variabilidad de un punto y un caso con una diferencia de dos puntos respecto al profesor. Esto nos puede indicar que tales diferencias se deban a las dificultades visibles en el uso del inglés con mayor frecuencia que en otros aspectos como la flexibilidad, la toma de responsabilidades, la participación activa, etcétera.

Con respecto a la coevaluación (COE) y la autocalificación (ACA), podemos observar que 37 casos coinciden exactamente en las puntuaciones. Presumimos que cuando se observan calificaciones idénticas, el equipo ha llegado a un acuerdo previo para calificarse de esa manera. Recordemos que la coevaluación debe realizarse fuera de clase y entregarla el día de la exposición. La consigna siempre ha sido la responsabilidad frente a las calificaciones para garantizar transparencia al proceso de enseñanza-aprendizaje. Este dato nos indica que $81.8 \%$ del alumnado ha puntuado diferente. En este transitar hacia un cambio en la forma de evaluar, este porcentaje nos demuestra que el alumnado, en su mayoría, ha acatado el cambio y que se ha atrevido a autoevaluarse y evaluar a sus pares. Podemos afirmar que el ejercicio de esta práctica en repetidas ocasiones puede contribuir al desarrollo de la objetividad y la autonomía del estudiantado en materia de evaluación.

También se puede observar que hay otros 37 casos en que la COE es superior a la ACA. En 31 casos la diferencia es de 0.5 puntos y seis casos con una diferencia de un punto. Deducimos primero que el nivel de autoexigencia es muy alto y, por ende, se tiende a puntuar menos a uno mismo y más al resto de compañeros; segundo, que el grado de presión y responsabilidad que gran parte del alumnado siente al calificar a otros se ve reflejado en puntuaciones más generosas, en ciertos casos para evitar malos entendidos entre sus pares. 


\section{Resultados de aprendizaje}

En este apartado se analizan las percepciones del estudiantado respecto a lo aprendido en la asignatura y se contrastará con la calificación numérica final obtenida. Según la Agencia Nacional de Evaluación de la Calidad y Acreditación (ANECA, 2013), los resultados de aprendizaje son las "declaraciones verificables de lo que un estudiante debe saber, comprender y ser capaz de hacer tras obtener una cualificación concreta, o tras culminar un programa o sus componentes". Durante el proceso se ha llevado un seguimiento de la evolución del alumnado, quien ha desarrollado tareas de manera individual y en equipo. Al finalizar la asignatura, y tras haber realizado la evaluación formativa y compartida, el alumnado responde a la pregunta abierta postratamiento: expresa tus opiniones acerca de la asignatura. Este ejercicio siempre nos resulta de gran utilidad puesto que arroja datos orientativos de las fortalezas y las debilidades en el planteamiento y el desarrollo de la asignatura. Por lo tanto, nos permite realizar los cambios pertinentes año tras ańo en busca de mejora y mayor adaptabilidad a la realidad del estudiantado.

Uno de los temas centrales que se identifican es la satisfacción por aprender la didáctica del inglés atendiendo a la secuencia de enseñanza propuesta. Recordemos que para facilitar una organización en el desarrollo de las clases de inglés se sugiere a los futuros maestros que introduzcan, de manera atrayente, el tema a tratar (en nuestro caso, estudiamos cómo adaptar y enseñar cuentos infantiles). Para ello, el alumnado debe apelar a los sentidos de los infantes, al gusto por descubrir, por adivinar (pre-telling).

Estudiar la didáctica aplicándola en clase me ha resultado de interés. No nos hemos quedado en el estudio teórico. El uso de recursos como los cambios de voz, la adaptación del aula y el mobiliario, y los recursos materiales han sido muy necesarios para comprender las formas de atraer la atención de los niños.

Me resultó divertido y grato jugar con los sentidos para enseńar los colores y sabores en el pre-teaching. De esa manera, los niños adquieren vocabulario que luego encontrarán en el cuento. 
Se ha proporcionado práctica y modelos para tratar la literatura en clases de preescolar y primaria (actual telling) como The Three Little Pigs, Cinderella o Little Red Riding Hood y sus versiones en formato musical por el grupo Debbie \& Friends. ${ }^{1}$ A su vez, se han diseñado actividades para reforzar lo aprendido (posttelling), como identificar elementos de los cuentos y nombrarlos, juegos interactivos, competiciones, reciclaje de vocabulario y expresiones mediante repetición y rutinas, música y danza con las innovaciones lingüísticas estudiadas, entre otros.

Muchos manifiestan haber mejorado en la fluidez con la que se expresan, siendo este uno de los resultados de aprendizaje más deseados por los docentes del área de inglés.

Siento que he hablado mucho en inglés. Esta asignatura me ha brindado mucha práctica oral y me ha servido para perder un poco el miedo a enfrentarme a una clase en inglés.

Me ha servido leer cuentos para poder después adaptarlos. De hecho, he aprendido vocabulario que nunca había visto. He utilizado actividades en mis prácticas.

Por otro lado, llama la atención que uno de los temas recurrentes sea la inseguridad para llevar a cabo la coevaluación. Una parte del alumnado manifiesta cierta incomodidad al evaluar a sus pares.

Creo que evaluar a los miembros del equipo ha generado malestar en algunos. No consideré correcto pactar la nota y siempre quise mantener el anonimato para evitar roces innecesarios. No todos estuvieron de acuerdo en principio. Para salir de dudas consultamos con la profesora quien nos recordó el carácter privado de la evaluación.

Si bien el estudiantado advierte que la coevaluación supone gran responsabilidad y posible tensión entre pares, esta actitud parece cambiar cuando se evalúan a sí mismos, tal como lo recoge el estudio cuantitativo del presente trabajo. Puede que

\footnotetext{
${ }^{1}$ Véase http://debbieandfriends.net/wp/kids-music-videos
} 
haya quien cuestione esta modalidad y se deduce que parte del alumnado no está lo suficientemente preparado para esta práctica porque no responde a un ejercicio de evaluación habitual en el ámbito académico. Otra razón de peso puede estar ligada a cierta inmadurez a la hora de responsabilizarse de la evaluación de otros. Recordemos que la puntuación que se otorga repercute en la nota final de asignatura. No obstante, por lo menos resulta interesante observar que, poco a poco, el alumnado se suma a esta propuesta haciendo un ejercicio responsable de su propia evaluación. Este es un primer paso en el camino a la consolidación de la autonomía, tal como lo plantea EEES y en la corroboración de los resultados de aprendizaje esperados.

Con respecto al rendimiento académico postratamiento alcanzado por el alumnado en la asignatura Teaching EFL and Children's Literature, la figura 2 recoge los resultados obtenidos. Se puede afirmar que el rendimiento académico en estos tres ańos es elevado, pues $95.5 \%$ supera la asignatura. La distribución de las calificaciones se mueve en los rangos del notable y el sobresaliente: $20 \%$ del alumnado alcanza el sobresaliente y $73.5 \%$ el notable. Los suspendidos alcanzan $4.4 \%$ del alumnado. Cabe aclarar que para este estudio solo se ha contado con el alumnado que asiste de manera regular a la asignatura, por tanto ha llevado a cabo la evaluación formativa y compartida.

FIGURA 2. Rendimiento académico postratamiento

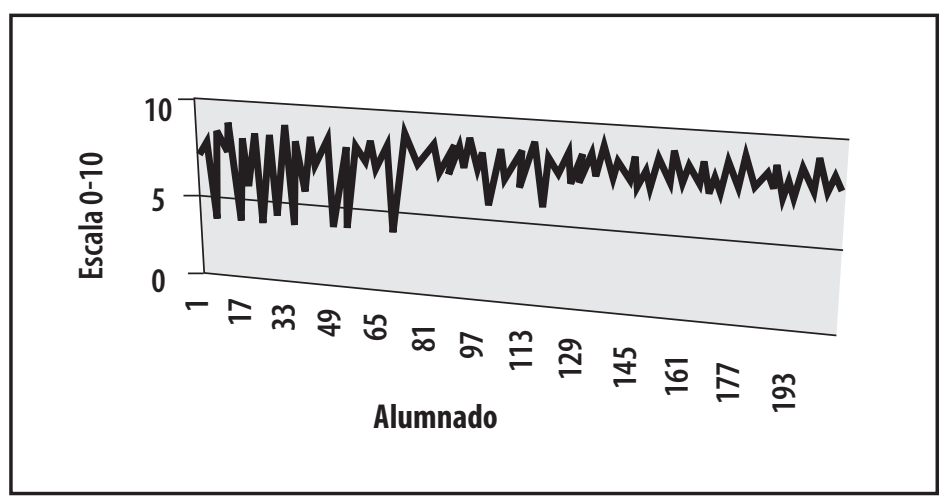


Si bien la propuesta de evaluación puede haber resultado compleja, los resultados de aprendizaje constatan que el estudiantado puede alcanzar calificaciones altas con el modelo de evaluación seguido, a la vez que logra regularse y adaptarse a las exigencias de la asignatura. Podemos inferir que se ha provisto de las herramientas necesarias para poder superar la asignatura de manera significativa.

\section{Aspectos mejorables}

\section{En relación con la evaluación}

El profesorado debería ser más taxativo sobre las indicaciones para llevar a cabo la coevaluación. Esta debe ser anónima y realizada fuera de clase de forma individual. De esta manera, cada miembro del equipo se responsabiliza de una evaluación más reflexionada y tal vez más justa.

Se desprende de algunas percepciones la dificultad de trabajar sincrónicamente con todos los miembros del equipo, hasta entonces un máximo de cinco alumnos. Hay quien sugiere un cambio para futuros cursos de un máximo de tres por equipo. Esta posibilidad va a ser contemplada ya que el profesorado concuerda que esta reducción aportaría mayor interacción entre los miembros, mayor coordinación y control de responsabilidades. Indefectiblemente, la evaluación formativa y compartida se puede ver beneficiada si se encuentra mayor armonía y conexión en los equipos.

\section{En relación con el trabajo en equipo}

Gran parte de los encuestados aluden a la falta de tiempo para poder llevar adelante los roles dentro de cada equipo de trabajo.

La propuesta ha sido muy interesante. He aprendido mucho de mis compañeros pero me ha resultado muy complicado realizar mis obligaciones como monitora lingüística, ya que he estado en periodo de prácticas en un colegio a la vez que cursando varias asignaturas. 
Las demandas de esta asignatura han sido un tanto elevadas. Me ha resultado difícil compatibilizar los horarios fuera de clase, el rol de secretaria y las prácticas. La preparación de material ha sido muy agotadora.

Como es de suponer, si bien el alumnado encuentra en el horario de clases un espacio para trabajar en sus proyectos, también es cierto que necesitan tiempo fuera de clase para reunirse. Sobre este aspecto, se ha propuesto una hoja de ruta donde cada equipo debe detallar el trabajo autónomo que realizará cada miembro. Se cuenta también con plataformas digitales como Google.docs y Edmodo para facilitar el trabajo, sin necesidad de desplazamientos. Se ha podido comprobar mucha interacción a través de estos medios que siempre han estado supervisados por el profesorado al momento de mantener las reuniones por equipo o individuales. Cabe destacar que al nivel lingüístico, estas plataformas sirven para hacer un uso activo de las destrezas lingüísticas al escribir y leer en inglés. El alumnado ha estado expuesto a la lengua inglesa más horas que en clases habituales de inglés por la carga de trabajo fuera de clase y la interacción en las plataformas educativas cuya lengua vehicular es el inglés.

Cabe reforzar en un futuro el uso asiduo de herramientas digitales para la elaboración de los proyectos y fomentar el debate y consenso sin necesidad de encontrar un espacio fuera de clase para reunirse. En muchos casos, resulta físicamente imposible puesto que el alumnado procede de distintos puntos de la comunidad valenciana y deben compatibilizar el horario de clases, sus prácticas en colegios con trabajos de otras asignaturas.

Por último, según se recoge de algunas opiniones, hay quienes proponen llevar la exposición de los proyectos a las escuelas de manera tal que el futuro maestro encuentre una conexión directa con lo que será su realidad inmediata. Esta práctica se ha venido haciendo en algunas aulas dentro de la universidad. Se ha invitado a escolares a las exposiciones de los cuentos en inglés y se ha comprobado un cambio de actitud muy positivo del futuro maestro y una mejora en su expresión oral en inglés al contar con niños como audiencia. 


\section{CONCLUSIONES}

Este trabajo parte de la necesidad de conocer las percepciones del alumnado respecto al modelo de evaluación propuesto para la asignatura Teaching EFL and Children's Literature y de presentar una serie de estrategias metodológicas para la aplicación de la evaluación formativa y compartida en la formación docente.

Sin duda, el rendimiento académico durante estos últimos tres años ha sido prominente, tanto como el nivel de participación activa del estudiantado frente a la propuesta de autoevaluación y coevaluación. Se recoge gran satisfacción respecto a esta modalidad de evaluación en general, así como ciertos aspectos cuestionados por algunos de los estudiantes. La inseguridad y tal vez incomodidad que genera evaluar a otros alumnos nos deja entrever una cierta inmadurez por parte de la población estudiantil, o bien una falta de costumbre respecto al acto. A pesar de ello, el estudiantado respondió de manera resolutiva en todo momento, y con la supervisión y orientación del profesorado se pudo llevar a cabo el proceso de evaluación de manera satisfactoria. Se deberá incidir más en años venideros sobre la importancia de las herramientas digitales de comunicación (Skype, Facetime, etc.) para evitar sobrecarga de trabajo y reuniones por equipos fuera de clase. Además, se deberá agrupar al alumnado por equipos de menor cantidad para garantizar mayor y mejor interacción entre las partes.

Respecto al uso del inglés como lengua vehicular y a la adquisición de técnicas y métodos didácticos para la enseñanza de la literatura en inglés, podemos resumir que la totalidad del alumnado manifiesta gran satisfacción con la propuesta de la asignatura, con la práctica ejercida y con la tutoría cercana por parte del profesorado.

Se puede afirmar que este modelo de seguimiento del proceso de formación articula todo proceso de enseñanza-aprendizaje. Tal y como sostienen Bordas y Cabrera (2001), una evaluación integradora incide cualitativamente en la calidad de la enseñanza y en la formación de la persona. Por lo tanto, podríamos determinar ciertos parámetros que pueden servir a los efectos de orientar la implementación de la evaluación formativa y compartida. Po- 
demos afirmar que un modelo de evaluación participativo debe plantearse como un ejercicio de responsabilidad frente al propio aprendizaje desde un primer momento, por tanto, la formación del profesorado debe estar orientada hacia un desempeño activo de reflexión y autoevaluación. En esta línea, la evaluación formativa y compartida se debería implementar en las aulas del profesorado en formación, con el fin de promover el desarrollo de la autocrítica y la formación de la persona. Cabe añadir que en los resultados de aprendizaje deben estar contempladas las competencias transversales que se persiguen con la implementación de la evaluación formativa y compartida. Por último, y en total acuerdo con Thousand, Villa y Nevin (2014), este modelo de evaluación debe garantizar una formación diferenciada del alumnado que fomente el diálogo y el seguimiento cercano de la evolución del estudiantado.

\section{REFERENCIAS BIBLIOGRÁFICAS}

Álvarez Valdivia, I. "La coevaluación como alternativa para mejorar la calidad del aprendizaje de los estudiantes universitarios: valoración de una experiencia”, en Revista Interuniversitaria de Formación del Profesorado, 22 (3), 2008, pp. 127-140.

Álvarez González, M. "La tutoría académica en el Espacio Europeo de la Educación Superior", en Revista interuniversitaria de formación del profesorado, 71, 2008.

Álvarez Méndez, J. La evaluación a examen. Ensayos criticos, Madrid, Miño y Dávila Editores, 2003.

Andreu Andrés, M A. y M. García Casas. "Evaluación, coevaluación y autoevaluación del trabajo en grupo en la lectura de mapas topográficos”, en F. Watts y A. García Carbonell (eds.). La evaluación compartida: investigación multidisciplinar, Valencia, Editorial UPV, 2006, pp. 131-164.

ANECA. Guía de apoyo para la redacción, puesta en práctica y evaluación de los resultados del aprendizaje. Versión 1.0. Madrid, 2013. Disponible en http://www.aneca.es/Documentos-y-publicaciones/Otros-documentos-de-interes/ 
Otras-guias-y-documentos-de-evaluacion/Guia-de-apoyo-para-la-redaccion-puesta-en-practica-y-evaluacion-delos-RESULTADOS-DEL-APRENDIZAJE.

Arias, C. y L. Maturana. "Evaluación en lenguas extranjeras: discursos y prácticas", en Íkala, Revista de lenguaje y cultura, 10 (16), 2005, pp. 63-91.

Arias, C., L. Maturana y M. Restrepo. "Evaluación de los aprendizajes en lenguas extranjeras: hacia prácticas justas y democráticas", en Lenguaje, 40 (1), 2012.

Biggs, J. "What the student does: teaching for enhanced learning", en Higher Education Research \& Development, 18 (1), 1999, pp. 57-75.

Biggs, J. Calidad del aprendizaje universitario, Madrid, Narcea, 2005.

Bonsón, M. y A. Benito. "Evaluación y aprendizaje”, en B. Águeda Benito y A. Cruz (eds.). Nuevas claves para la docencia universitaria en el Espacio Europeo de Educación Superior, Madrid, Narcea, 2005, pp. 87-100.

Bordas, I. y F. Cabrera. "Estrategias de evaluación de los aprendizajes centrados en el proceso", en Revista Española de Pedagogía, 218, 2001, pp. 25-48.

Boud, D. y N. Falchikov. Rethinking assessment in higher education: Learning for the longer term, Nueva York, Routledge, 2007.

Bretones Román, A. "Las preconcepciones del estudiante de profesorado: de la construcción y transmisión del conocimiento a la participación en el aula”, en Educar (32), 2003, pp. 25-54.

Brown, S. y A. Glasner. Evaluar en la universidad: problemas y nuevos enfoques, Madrid, Narcea Ediciones, 2003.

Casasempere, A. Inmigración y educación, Curso Atlas.ti, Valencia, UPV, 2007.

Castejón Oliva, F., V. López Pastor, J. Julián Clemente y J. Zaragoza Casterad. "Evaluación formativa y rendimiento académico en la formación inicial del profesorado de educación física”, en International Journal of Medicine and Science of Physical Activity and Sport, 11 (42), 2011, pp. 238-346. 
Cebrián de la Serna, M., J. Serrano Angulo y M. Ruiz Torres. "eRubrics in Cooperative Assessment of Learning at University”, en Comunicar, 22 (43), 2014, pp. 153-161.

Charmaz, K. Constructing Grounded Theory. A Practical Guide through Qualitative Analysis, Londres, Sage, 2006.

Contreras, G. "Evaluación formativa. Nuevos enfoques. Pensamiento Educativo", en Revista de Investigación Educacional Latinoamericana, 33 (2), 2011, pp. 31-48.

De la Fuente, J. y F. Justicia. "Regulación de la enseñanza para la autorregulación del aprendizaje en la Universidad", en Aula abierta, 82, 2003, pp. 161-171.

De Miguel Díaz, M. "Metodologías para optimizar el aprendizaje. Segundo objetivo del Espacio Europeo de Educación Superior", en Revista interuniversitaria de formación del profesorado, 57, 2006, pp. 71-92.

Delgado García, A., R. Borge Bravo, J. García Albero, R. Oliver Cuello y L. Salomón Sancho. "Competencias y diseño de la evaluación continua y final en el Espacio Europeo de Educación Superior”, Programa de Estudios y análisis de la Dirección General de Universidades del Ministerio de Educación y Ciencia, 2005. Disponible en http://campus.usal.es/ - ofeees/ARTICULOS/competencias_evaluacion_eees_mec.pdf

Dochy, F., M. Segers y S. Dierick. "Nuevas vías de aprendizaje y enseñanza y sus consecuencias: una nueva era de evaluación”, en Revista de Docencia universitaria, 2 (2), 2002.

Fernández March, A. "La evaluación orientada al aprendizaje en un modelo de formación por competencias en la educación universitaria", en REDU. Revista de Docencia Universitaria, 8 (1), 2010.

Freire, P. La naturaleza politica de la educación: cultura, poder y liberación, Barcelona, Paidós-MEC, 1990.

Gargallo López, B., G. Almerich Cerveró, J. Giménez Beut, C. Campos Aparicio y B. Sarriá Chust. "La evaluación de enfoques y estrategias de aprendizaje en estudiantes universitarios", en XII Jornadas de Redes de investigación en Docencia Universitaria, Universidad de Alicante, s/f, ISBN: 2014978-84-607-0709-8. 
Glaser, B. y A. Strauss. Discovery of Grounded Theory. Strategies for Qualitative Research, Nueva York, Aldine Publishing Company, 1967.

González Pérez, M. "La evaluación del aprendizaje: tendencias y reflexión crítica”, en Educación Media Superior, 15 (1), 2001, pp. 85-96.

Hannafin, M. "Student-centered learning", en Encyclopedia of the Sciences of Learning, Nueva York, Springer, 2012, pp. 3211-3214.

Hannafin, M., J. Hill, J., S. Land y E. Lee. "Student-centered, open learning environments: research, theory, and practice", en Handbook of Research on Educational Communications and Technology, Nueva York, Springer, 2014, pp. 641-651.

Klenowski, V. Desarrollo del portafolio para el aprendizaje y la evaluación: procesos y principios, Madrid, Narcea Ediciones, 2005.

Knight, P. El profesorado de Educación Superior, Madrid, Narcea, 2005.

Kolb, D. y A. Kolb. The Kolb Learning Style Inventory. Version 3.1, 2005 Technical Specifications, Boston, Hay Group, Hay Resources Direct, 2005a.

Kolb, A. y D. Kolb. "Learning styles and learning spaces: Enhancing experiential learning in higher education", en Academy of Management Learning and Education, 4 (2), 2005b, pp. 193-212.

Lamas Rojas, H. "Aprendizaje autorregulado, motivación y rendimiento académico", en Liberabit, 14 (14), 2008, pp. $15-20$.

López, A. A. "La evaluación formativa en la enseñanza y aprendizaje del inglés", en Voces y Silencios, 1 (2), 2010, pp. 111-124.

López Pastor, V. "La participación del alumnado en los procesos evaluativos: la autoevaluación y la evaluación compartida en educación física”, en A. Fraile Aranda (ed.). Didáctica de la Educación Física: una perspectiva crítica y transversal, Madrid, Biblioteca Nueva, 2004, pp. 265-290. 
López Pastor, V., M. González Pascual y J. Barba Martín. “La participación del alumnado en la evaluación: la autoevaluación, la coevaluación y la evaluación compartida", en Tandem. Didáctica de la Educación Física, 17, 2005, pp. 21-37.

López Pastor, V. "El papel de la evaluación formativa en el proceso de convergencia hacia el EEES. Análisis del estado de la cuestión y presentación de un sistema de intervención", en Revista Interuniversitaria de Formación del Profesorado, 20 (3), 2006, pp. 93-119.

López Pastor, V. et al. "Trece años de evaluación compartida en Educación Física", en Revista Internacional de Medicina y Ciencias de la Actividad Fisica y el Deporte, 7 (26) 2007, pp. 69-86.

López Pastor, V. "Desarrollando sistemas de evaluación formativa y compartida en la docencia universitaria. Análisis de resultados de su puesta en práctica en la formación inicial del profesorado", en European Journal of Teacher Education, 31 (3), 2008, pp. 293-311.

López Pastor, V. Evaluación formativa y compartida en Educación Superior: propuestas, técnicas, instrumentos y experiencias, Madrid, Narcea Ediciones, 2009.

López Pastor, V., J. Fernández Balboa, M. Santos Pastor y A. Fraile Aranda. 'Students' self-grading, professor's grading and negotiated final grading at three university programmes: analysis of reliability and grade difference ranges and tendencies", en Assessment \& Evaluation in Higher Education, 37 (4), 2012, pp. 453-464.

Martínez-Muñoz, F., M. Santos Pastor y A. Sicilia. "De la pedagogía del silencio a la pedagogía del diálogo. La autoevaluación y la autocalificación como formas de promoción democrática”, en V. López Pastor (ed.). La Evaluación en Educación Física. Revisión de los modelos tradicionales y planteamiento de una alternativa: la evaluación formativa y compartida, Buenos Aires, Miñó y Dávila, 2006.

Martínez, L., M. Martín y M. Capllonch. "Una experiencia de desarrollo profesional del docente universitario de Educación Física a través de una práctica crítica, reflexiva y 
colaborativa”, en Cultura y Educación, 21 (1), 2009, pp. 95-106.

Martínez Rizo, F. "Evaluación formativa en aula y evaluación a gran escala: hacia un sistema más equilibrado", en Revista electrónica de investigación educativa, 11 (2), 2009, pp. $1-18$.

Martinic, S. et al. "Análisis comparativo del componente de práctica en el currículo de formación profesional de médicos y profesores en la Pontificia Universidad Católica de Chile”, en Estudios Pedagógicos, 40 (1), 2014, pp. 179-196.

Marshall, B. y M. Drummond. "How teachers engage with assessment for learning: Lessons from the classroom", en Research papers in education, 21 (02), 2006, pp. 133149.

Matthews, C. y J. Marino. Professional Interaction: Oral Communication Skills in Science, Technology and Medicine, Nueva Jersey, Prentice Hall, 1990.

Mérida-Serrano, R. "Nueva percepción de la identidad profesional del docente universitario ante la convergencia europea”, en Revista Electrónica de Investigación Educativa, 8 (1), 2006.

Morales, P. "Investigación e innovación educativa", en REICE: Revista Electrónica Iberoamericana sobre Calidad, Eficacia y Cambio en Educación, 8 (2), 2010, pp. 47-73.

$\mathrm{Na}, \mathrm{Z}$. "A study of high school students' English learning anxiety", en The Asian EFL Journal, 9 (3), 2007, pp. 22-34.

Perassi, Z. La evaluación en educación: un campo de controversias, Argentina, LAE-Laboratorio de Alternativas Educativas, 2008.

Pérez Pueyo, A. et al. "Evaluación formativa y compartida en la docencia universitaria y el Espacio Europeo de Educación Superior: cuestiones clave para su puesta en práctica”, en Revista de Educación, 347, 2008, pp. 435-451.

Restrepo, A. y H. Nelson. "Role of Systematic Formative Assessment on Students' Views of Their Learning”, en Profile, Issues in Teachers' Professional Development, 15 (2), 2013. 
Reynolds, C. R., R. L. Livingston y V. L. Willson. Measurement and assessment in in education, Boston, Pearson, 2006.

Rice, P. Making Learning Happen. A guide for post-compulsory education, Londres, Sage, 2014.

Rodríguez Gómez, G., S. Ibarra Sáiz y E. García Jiménez. “Autoevaluación, evaluación entre iguales y coevaluación: conceptualización y práctica en las universidades españolas”, en Revista de investigación en educación, 2 (11), 2013, pp. 198-210.

Rodríguez Mena García, M. e I. García-Montero. El aprendizaje para el cambio: papel de la educación, México, Universidad Autónoma del Estado de México, 2003.

Somervell, H. "Issues in assessment, enterprise and higher education: The case for self-peer and collaborative assessment", en Assessment and evaluation in Higher Education, 18 (3), 1993, pp. 221-233.

Thousand, S., R. Villa y A. Nevin (eds.). Differentiating Instruction: Planning for Universal Design and Teaching for College and Career Readiness, Londres, Corwin Press, 2014.

Villardón Gallego, L. "Evaluación del aprendizaje para promover el desarrollo de competencias", en Educación siglo XXI, 24, 2006, pp. 57-76.

Watts, F. y A. García Carbonell, A. La evaluación compartida: investigación multidisciplinar, Valencia, Universidad Politécnica de Valencia, 2006.

Watts, F., A. García Carbonell y N. Martínez Alzamora. "Relación entre autoevaluación y evaluación por el profesor. Estimación del tiempo de dedicación del estudiante", en F. Watts y A. García-Carbonell (eds.). La evaluación compartida: investigación multidisciplinar, Valencia, Editorial UPV, 2006, pp. 131-164.

Weinstein, C. E., T. W. Acee y J. Jung. "Self-regulation and learning strategies" en New Directions for Teaching and Learning, (126), 2011, pp. 45-53.

Wen-ming, Y. "On the Developing Trend of the College English Assessment", en Journal of Taiyuan University of Technology (Social Sciences Edition), 2, 2003, pp. 1-26. 
Yi'An, W. "English language teaching in China: Trends and challenges", en TESOL Quarterly, 35 (1) 2001, pp. 191-194.

Zabalza Beraza, M. La enseñanza universitaria: el escenario y sus protagonistas, Madrid, Narcea Ediciones, 2002.

Zabalza Beraza, M. Competencias docentes del profesorado universitario: calidad y desarrollo profesional, Madrid, Narcea Ediciones, 2003.

Zaragoza Casterad, J., J. Luis Pascual y J. Manrique Arribas. "Experiencias de innovación en docencia universitaria: resultados de la aplicación de sistemas de evaluación formativa", en REDU. Revista de Docencia Universitaria, 7 (4), 2009, pp. 1-33.

Zhi-qiang, Y. "Formative Assessment and College English Teaching and Testing" en Research on Education Tsinghua University, 5, 2003, pp. 1-18.

Zou, H. M. y Z. Cai. "An Experiment on Implementation of Formative Assessment and the Reform of the Current Assessing System of College English Teaching", en Journal of Jiangxi Normal University (Philosophy and Social Sciences Edition), 39 (1), 2006, pp. 114-118. 